\title{
Growth of Logarithmic Derivatives and Their Applications in Complex Differential Equations
}

\author{
Zinelâabidine Latreuch and Benharrat Belaïdi \\ Department of Mathematics, Laboratory of Pure and Applied Mathematics, University of Mostaganem (UMAB), \\ B. P. 227, Mostaganem, Algeria
}

Correspondence should be addressed to Benharrat Belaïdi; belaidi@univ-mosta.dz

Received 29 November 2013; Accepted 21 January 2014; Published 4 March 2014

Academic Editor: Chuanxi Qian

Copyright (C) 2014 Z. Latreuch and B. Belaïdi. This is an open access article distributed under the Creative Commons Attribution License, which permits unrestricted use, distribution, and reproduction in any medium, provided the original work is properly cited.

We continue the study of the behavior of the growth of logarithmic derivatives. In fact, we prove some relations between the value distribution of solutions of linear differential equations and growth of their logarithmic derivatives. We also give an estimate of the growth of the quotient of two differential polynomials generated by solutions of the equation $f^{\prime \prime}+A(z) f^{\prime}+B(z) f=0$, where $A(z)$ and $B(z)$ are entire functions.

\section{Introduction and Main Results}

Throughout this paper, we assume that the reader is familiar with the fundamental results and the standard notations of Nevanlinna's value distribution theory (see [1-4]). In addition, we will denote by $\lambda(f)$ (resp., $\bar{\lambda}(f))$ and $\lambda(1 / f)$ (resp., $\bar{\lambda}(1 / f)$ ) the exponent of convergence of zeros (resp., distinct zeros) and poles (resp., distinct poles) of a meromorphic function $f, \rho(f)$ to denote the order of growth of $f$. A meromorphic function $\varphi(z)$ is called a small function with respect to $f(z)$ if $T(r, \varphi)=o(T(r, f))$ as $r \rightarrow+\infty$ except possibly a set of $r$ of finite linear measure, where $T(r, f)$ is the Nevanlinna characteristic function of $f$.

Definition 1 (see [4]). Let $f$ be a meromorphic function. Then the hyperorder of $f(z)$ is defined as

$$
\rho_{2}(f)=\limsup _{r \rightarrow+\infty} \frac{\log \log T(r, f)}{\log r} .
$$

Definition 2 (see $[1,3])$. The type of a meromorphic function $f$ of order $\rho(0<\rho<\infty)$ is defined as

$$
\tau(f)=\limsup _{r \rightarrow+\infty} \frac{T(r, f)}{r^{\rho}} .
$$

If $f$ is an entire function, then the type of $f$ of order $\rho(0<$ $\rho<\infty)$ is defined as

$$
\tau_{M}(f)=\limsup _{r \rightarrow+\infty} \frac{\log M(r, f)}{r^{\rho}},
$$

where $M(r, f)=\max _{|z|=r}|f(z)|$ is the maximum modulus function.

Remark 3. For entire function, we can have $\tau_{M}(f) \neq \tau(f)$. For example, if $f(z)=e^{z}$, then we have $\tau_{M}(f)=1$ and $\tau(f)=$ $1 / \pi$.

Growth of logarithmic derivative of meromorphic functions has been generously considered during the last decades, among others, by Gol'dberg and Grinšteĭn [5], Benbourenane and Korhonen [6], Hinkkanen [7], and Heittokangas et al. [8]. All these considerations have been devoted to getting more detailed estimates to the proximity function $m\left(r, f^{\prime} / f\right)$ than what is the original Nevanlinna estimate that essentially can be written as $m\left(r, f^{\prime} / f\right)=S(r, f)$. The same applies for the case of higher logarithmic derivatives $m\left(r, f^{(k)} / f\right)$ as well. The estimation of logarithmic derivatives plays the key role in theory of differential equations. In his paper Gundersen [9] proved some interesting inequalities on the module of logarithmic derivatives of meromorphic functions. 
Recently [10], the authors have studied some properties of the behavior of growth of logarithmic derivatives of entire and meromorphic functions and have obtained some relations between the zeros of entire functions and the growth of their logarithmic derivatives. In fact, they have proved the following.

Theorem A (see [10]). Let $f$ be an entire function with finite number of zeros. Then, for any integer $k \geq 1$,

$$
\rho\left(\frac{f^{(k)}}{f}\right)=\rho_{2}(f) .
$$

Theorem B (see [10]). Suppose that $k \geq 2$ is an integer and let $f$ be a meromorphic function. Then

$$
\begin{aligned}
\rho\left(\frac{f^{\prime}}{f}\right) & =\max \left\{\rho\left(\frac{f^{(k)}}{f}\right), k \geq 2\right\} \\
& =\max \left\{\rho\left(\frac{f^{(k)}}{f}\right), \rho\left(\frac{f^{(k+1)}}{f}\right), k \geq 2\right\} .
\end{aligned}
$$

In [11, pages 457-458], Bank and Langley have obtained the following result.

Theorem C (see [11]). All nontrivial solutions of

$$
f^{\prime \prime}+e^{P(z)} f=0
$$

where $P(z)$ is a nonconstant polynomial, satisfy $\lambda(f)=\infty$.

Theorem D (see [4] (E. Picard)). Any transcendental entire function $f$ must take every finite complex value infinitely many times, with at most one exception.

Example 4. The entire function $e^{z}$ omits the value zero and every other finite value is assumed infinitely often and zero is the exceptional value of Picard.

Question 1. Under what conditions can we ensure that $f$ has no Picard's exceptional value?

Question 2. Under what conditions can we obtain the same result as Theorem $\mathrm{C}$ for higher order linear differential equations with entire coefficients?

The subject of this paper is to give answers to the above questions. In fact, we prove some relations between the value distribution of solutions of linear differential equations and growth of their logarithmic derivatives. Firstly, we study the relationship between the growth of logarithmic derivatives of solutions of complex differential equation

$$
f^{(k)}+A_{k-1} f^{(k-1)}+\cdots+A_{0} f=0
$$

and their exponents of convergence. We will also prove the nonexistence of any Picard exceptional value of solutions of (7), and we obtain the following.

Theorem 5. Let $A_{0}, A_{1}, \ldots, A_{k-1}$ be entire functions satisfying $\rho\left(A_{0}\right)=\rho(0<\rho<\infty), \tau_{M}\left(A_{0}\right)=\tau(0<\tau<\infty)$, and let $\rho\left(A_{j}\right) \leq \rho, \tau_{M}\left(A_{j}\right)<\tau$ if $\rho\left(A_{j}\right)=\rho(j=1, \ldots, k-1)$. If $f$ is a nontrivial solution of $(7)$, such that $\rho\left(f^{\prime} / f\right)>\rho\left(A_{0}\right)$, then $f$ assume every finite complex value infinitely often.

Furthermore, if $\rho\left(f^{\prime} / f\right)=\infty$, then

$$
\bar{\lambda}(f)=\lambda(f)=\infty .
$$

Corollary 6. Under the hypotheses of Theorem 5, let $\varphi(z)$ be an entire function with finite order. If $f$ is a nontrivial solution of (7), such that $\rho\left(f^{(j+1)} / f^{(j)}\right)=\infty$ for some $j \in \mathbb{N}$, then

$$
\bar{\lambda}\left(f^{(j)}-\varphi\right)=\lambda\left(f^{(j)}-\varphi\right)=\infty .
$$

Corollary 7. Under the hypotheses of Theorem 5, every nontrivial solution of (7) does not have any Picard exceptional values.

Example 8. It is clear that the function $f(z)=\sin e^{z}$ satisfies the differential equation

$$
f^{\prime \prime}-f^{\prime}+e^{2 z} f=0
$$

and $\rho\left(f^{\prime} / f\right)=\rho(f)=\infty$. Obviously, the conditions of Theorem 5 are satisfied. Then $\bar{\lambda}(f)=\lambda(f)=\infty$.

Secondly, we consider the differential equation

$$
f^{\prime \prime}+A(z) f^{\prime}+B(z) f=0
$$

where $A(z)$ and $B(z)$ are entire functions, and let $P_{n}[f]$ and $Q_{m}[f]$ be two differential polynomials defined by

$$
\begin{gathered}
P_{n}[f]=d_{n} f^{(n)}+d_{n-1} f^{(n-1)}+\cdots+d_{0} f \quad(n \geq 1), \\
Q_{m}[f]=b_{m} f^{(m)}+b_{m-1} f^{(m-1)}+\cdots+b_{0} f \quad(m \geq 1),
\end{gathered}
$$

where $d_{i}(i=0, \ldots, n)$ and $b_{j}(j=0, \ldots, m)$ are entire functions not all vanishing identically. In recent years, there is a great interest to investigate the growth and oscillation of differential polynomials generated by solutions of differential equations in the complex plane (see [12-14]). In this part, we give under some conditions an estimate on the growth of the quotient of two differential polynomials generated by solutions of (11), and we obtain the following results.

Theorem 9. Let $f$ be a nontrivial solution of (11), and let $d_{i}(i=0, \ldots, n)$ and $b_{j}(j=0, \ldots, m)$ be entire functions not all vanishing identically such that $g_{f}=P_{n}[f] / Q_{m}[f]$ is irreducible function in $f^{\prime} / f$. If

$$
\begin{array}{r}
\max \left\{\rho(A), \rho(B), \rho\left(d_{i}\right)(i=0, \ldots, n),\right. \\
\left.\rho\left(b_{j}\right)(j=0, \ldots, m)\right\}<\rho\left(\frac{f^{\prime}}{f}\right),
\end{array}
$$

then

$$
\rho\left(g_{f}\right)=\rho\left(\frac{f^{\prime}}{f}\right) .
$$


Remark 10. In Theorem 9, the condition (13) is necessary. For example, $f(z)=\exp \left(e^{z}\right)$ is a solution of the differential equation

$$
f^{\prime \prime}-\left(e^{z}+e^{2 z}\right) f=0
$$

with

$$
\rho\left(\frac{f^{\prime}}{f}\right)=1
$$

and let

$$
g_{f}=\frac{d_{2} f^{\prime \prime}+d_{1} f^{\prime}+d_{0} f}{b_{2} f^{\prime \prime}+b_{1} f^{\prime}+b_{0} f}=\frac{d_{2}\left(e^{z}+e^{2 z}\right)+d_{1} e^{z}+d_{0}}{b_{2}\left(e^{z}+e^{2 z}\right)+b_{1} e^{z}+b_{0}}
$$

If we take $d_{2}=e^{z^{2}}, d_{1}=d_{0}=1$, and $b_{2}=b_{1}=b_{0}=1$, then

$$
\rho\left(g_{f}\right)=2>\rho\left(\frac{f^{\prime}}{f}\right)=1 \text {. }
$$

Remark 11. In Theorem 9, the condition that $g_{f}$ is irreducible function in $f^{\prime} / f$ is necessary. If $d_{i}=b_{j}(n=m)$, then $g_{f} \equiv 1$ and so

$$
\rho\left(g_{f}\right)=0 \neq \rho\left(\frac{f^{\prime}}{f}\right)
$$

By setting $n=m=2$ in Theorem 9, we can obtain the following special case.

Corollary 12. Let $A(z)$ and $B(z)$ be entire functions of finite order such that $\rho(A)<\rho(B)$ and $0<\tau(A)<\tau(B)<\infty$ if $\rho(B)=\rho(A)>0$. Let $d_{i}(z)(i=0,1,2), b_{j}(z)(j=0,1,2)$ be entire functions that are not all vanishing identically such that

$$
\max \left\{\rho\left(d_{i}\right), \rho\left(b_{j}\right), 0 \leq i, j \leq 2\right\}<\rho(B)
$$

and $d_{2}(z) b_{1}(z)-d_{1}(z) b_{2}(z) \neq \equiv$. If $f \neq \equiv 0$ is a solution of (11), such that $\rho(B)<\rho\left(f^{\prime} / f\right)$, then

$$
\rho\left(\frac{d_{2} f^{\prime \prime}+d_{1} f^{\prime}+d_{0} f}{b_{2} f^{\prime \prime}+b_{1} f^{\prime}+b_{0} f}\right)=\rho\left(\frac{f^{\prime}}{f}\right) .
$$

Corollary 13. Under the hypotheses of Corollary 12, if $f$ is a nontrivial solution of (11), such that $\rho\left(f^{\prime} / f\right)=\infty$, then

$$
\bar{\lambda}(f)=\bar{\lambda}\left(f^{\prime}\right)=\infty \text {. }
$$

\section{Auxiliary Lemmas}

Lemma 14 (see [1]). Let $f$ be a meromorphic function and let $k \in \mathbb{N}$. Then

$$
m\left(r, \frac{f^{(k)}}{f}\right)=S(r, f)
$$

where $S(r, f)=O(\log T(r, f)+\log r)$, possibly outside a set $E \subset(0, \infty)$ with a finite linear measure. If $f$ is a finite order of growth, then

$$
m\left(r, \frac{f^{(k)}}{f}\right)=O(\log r)
$$

Lemma 15 (see [15]). Let $A_{0}(z), \ldots, A_{k-1}(z)$ be entire functions of finite order such that

$$
\max \left\{\rho\left(A_{j}\right): j=1, \ldots, k-1\right\}<\rho\left(A_{0}\right) .
$$

Then every solution $f \not \equiv 0$ of (7) satisfies $\rho(f)=+\infty$ and $\rho_{2}(f)=\rho\left(A_{0}\right)$.

Lemma 16 (see [16]). Let $A_{0}, A_{1}, \ldots, A_{k-1}$ be entire functions satisfying $\rho\left(A_{0}\right)=\rho(0<\rho<\infty), \tau_{M}\left(A_{0}\right)=\tau(0<\tau<\infty)$, and let $\rho\left(A_{j}\right) \leq \rho, \tau_{M}\left(A_{j}\right)<\tau$ if $\rho\left(A_{j}\right)=\rho(j=1, \ldots, k-1)$. Then every solution $f \neq 0$ of $(7)$ satisfies $\rho(f)=+\infty$ and $\rho_{2}(f)=\rho\left(A_{0}\right)$.

Here, we give a special case of the result given by Xu et al. in [17].

Lemma 17. Let $A_{0}, A_{1}, \ldots, A_{k-1}$ be entire functions with finite order and let them satisfy one of the following conditions:

(i) $\max \left\{\rho\left(A_{j}\right): j=1, \ldots, k-1\right\}<\rho\left(A_{0}\right)<\infty$;

(ii) $0<\rho\left(A_{k-1}\right)=\cdots=\rho\left(A_{0}\right)<\infty$ and $\max \left\{\tau_{M}\left(A_{j}\right)\right.$ : $j=1, \ldots, k-1\}<\tau_{M}\left(A_{0}\right)<\infty$.

Then, for every solution $f \not \equiv \quad 0$ of (7) and for any entire function $\varphi(z)$ 三 0 satisfying $\rho(\varphi)<\infty$, we have

$$
\begin{gathered}
\bar{\lambda}\left(f^{(j)}-\varphi\right)=\lambda\left(f^{(j)}-\varphi\right)=\rho(f)=\infty \quad(j \in \mathbb{N}), \\
\bar{\lambda}_{2}\left(f^{(j)}-\varphi\right)=\lambda_{2}\left(f^{(j)}-\varphi\right)=\rho\left(A_{0}\right) \quad(j \in \mathbb{N}) .
\end{gathered}
$$

Lemma 18 (see [10]). Let $f$ be a meromorphic function. If there exists an integer $k \geq 1$, such that $\rho\left(f^{(k)} / f\right)=\rho(f)$ and $\rho(f)>\rho_{2}(f)$, then

$$
\max \left\{\bar{\lambda}(f), \bar{\lambda}\left(\frac{1}{f}\right)\right\}=\max \left\{\lambda(f), \lambda\left(\frac{1}{f}\right)\right\}=\rho(f) .
$$

Furthermore, if $f$ is entire function, then

$$
\bar{\lambda}(f)=\lambda(f)=\rho(f) .
$$

By the principle of mathematical induction we can obtain easily the following lemma.

Lemma 19. Suppose that $f^{\prime \prime}=-A(z) f^{\prime}-B(z) f$, where $A(z)$ and $B(z)$ are entire functions. Then, for an integer $j \geq 2$, there exist entire functions $\alpha_{j}$ and $\beta_{j}$ such that

$$
f^{(j)}=\alpha_{j} f^{\prime}+\beta_{j} f
$$


Lemma 20 (see [18]). Let $f$ be a solution of (7) where the coefficients $A_{j}(z)(j=0, \ldots, k-1)$ are analytic functions in the $\operatorname{disc} \Delta_{R}=\{z \in \mathbb{C}:|z|<R\}, 0<R \leq \infty$. Let $n_{c} \in\{1, \ldots, k\}$ be the number of nonzero coefficients $A_{j}(z)(j=0, \ldots, k-1)$, and let $\theta \in\left[0,2 \pi\left[\right.\right.$ and $\varepsilon>0$. If $z_{\theta}=v e^{i \theta} \in \Delta_{R}$ such that $A_{j}\left(z_{\theta}\right) \neq 0$ for some $j=0, \ldots, k-1$, then, for all $\nu<r<R$,

$$
\left|f\left(r e^{i \theta}\right)\right| \leq C \exp \left(n_{c} \int_{v}^{r} \max _{j=0, \ldots, k-1}\left|A_{j}\left(t e^{i \theta}\right)\right|^{1 /(k-j)} d t\right),
$$

where $C>0$ is a constant satisfying

$$
C \leq(1+\varepsilon) \max _{j=0, \ldots, k-1}\left(\frac{\left|f^{(j)}\left(z_{\theta}\right)\right|}{\left(n_{c}\right)^{j} \max _{n=0, \ldots, k-1}\left|A_{n}\left(z_{\theta}\right)\right|^{j /(k-n)}}\right) .
$$

Here, we give a special case of the result given by Cao et al. in [19].

Lemma 21. Let $f$ be a meromorphic function with finite order $0<\rho(f)<\infty$ and type $0<\tau(f)<\infty$. Then for any given $\beta<\tau(f)$ there exists a subset I of $[1,+\infty)$ that has infinite logarithmic measure such that $T(r, f)>\beta r^{\rho(f)}$ holds for all $r \in I$.

Lemma 22. Let $A(z)$ and $B(z)$ be entire functions such that $\rho(B)=\rho(0<\rho<\infty), \tau(B)=\tau(0<\tau<\infty)$, and let $\rho(A)<$ $\rho(B)$ and $\tau(A)<\tau(B)$ if $\rho(A)=\rho(B)$. If $f \neq \equiv 0$ is a solution of

$$
f^{\prime \prime}+A(z) f^{\prime}+B(z) f=0
$$

then $\rho(f)=\infty$ and $\rho_{2}(f)=\rho(B)$.

Proof. If $\rho(A)<\rho(B)$, then, by Lemma 15, we obtain the result. We prove only the case when $\rho(A)=\rho(B)=\rho$ and $\tau(A)<\tau(B)$. Since $f \not \equiv 0$, then, by (32), we have

$$
B=-\left(\frac{f^{\prime \prime}}{f}+A \frac{f^{\prime}}{f}\right) \text {. }
$$

Suppose that $f$ is of finite order; then by (33) and Lemma 14 we obtain

$$
T(r, B) \leq T(r, A)+O(\log r)
$$

which implies the contradiction

$$
\tau(B) \leq \tau(A) .
$$

Hence $\rho(f)=\infty$. By using inequality (30) for $R=\infty$, we have

$$
\rho_{2}(f) \leq \max \{\rho(A), \rho(B)\}=\rho(B) .
$$

On the other hand, since $\rho(f)=\infty$, then, by (33) and Lemma 14,

$$
T(r, B) \leq T(r, A)+S(r, f)
$$

where $S(r, f)=O(\log T(r, f)+\log r)$, possibly outside a set $E \subset(0,+\infty)$ with a finite linear measure. By $\tau(A)<\tau(B)$ we choose $\alpha_{0}, \alpha_{1}$ satisfying $\tau(A)<\alpha_{1}<\alpha_{0}<\tau(B)$ such that, for $r \rightarrow+\infty$, we have

$$
T(r, A) \leq \alpha_{1} r^{\rho}
$$

By Lemma 21, there exists a subset $E_{1} \subset[1,+\infty)$ of infinite logarithmic measure such that

$$
T(r, B)>\alpha_{0} r^{\rho} .
$$

By (37)-(39) we obtain, for all $r \in E_{1} \backslash E$,

$$
\left(\alpha_{0}-\alpha_{1}\right) r^{\rho} \leq O(\log T(r, f))+O(\log r)
$$

which implies

$$
\rho(B)=\rho \leq \rho_{2}(f) .
$$

By using (36) and (41), we obtain $\rho_{2}(f)=\rho(B)$.

Remark 23. Lemma 22 was obtained by Tu and Yi in [16] for higher order linear differential equations by using the type $\tau_{M}$ (Lemma 16 in the present paper).

Lemma 24 (see [20]). Let $f$ and $g$ be meromorphic functions such that $0<\rho(f), \rho(g)<\infty$ and $0<\tau(f), \tau(g)<\infty$. Then we have the following.

(i) If $\rho(f)>\rho(g)$, then we obtain

$$
\tau(f+g)=\tau(f g)=\tau(f) .
$$

(ii) If $\rho(f)=\rho(g)$ and $\tau(f) \neq \tau(g)$, then we get

$$
\rho(f+g)=\rho(f g)=\rho(f)=\rho(g) .
$$

\section{Proof of Theorems and Corollaries}

Proof of Theorem 5. By the conditions of Theorem 5 and Lemma 16, every nontrivial solution $f$ of (7) is of infinite order and $\rho_{2}(f)=\rho\left(A_{0}\right)$. We need to prove that every solution $f$ attains every complex value without exception; that is, for all $a \in \mathbb{C}$, the equation $f(z)=a$ has infinitely many solutions. We have two cases

(i) If $a \neq 0$, then, by Lemma 17, we have $\lambda(f-a)=$ $+\infty$ and the equation $f(z)=a$ has infinitely many solutions.

(ii) If $a=0$, then we suppose that $f$ has a finite number of zeros. By Theorem A

$$
\rho\left(A_{0}\right)<\rho\left(\frac{f^{\prime}}{f}\right)=\rho_{2}(f)=\rho\left(A_{0}\right)
$$

which is a contradiction, and hence $f$ assume every finite complex value infinitely often. Now, if $\rho\left(f^{\prime} / f\right)=\rho(f)=\infty$ and since $\rho_{2}(f)=\rho\left(A_{0}\right)<\rho(f)=\infty$, then by using Lemma 18 we have

$$
\bar{\lambda}(f)=\lambda(f)=\rho(f)=\infty .
$$


Proof of Corollary 6. By using Lemma 17, we have for any finite order entire function $\varphi(z) \neq \equiv$

$$
\bar{\lambda}\left(f^{(j)}-\varphi\right)=\lambda\left(f^{(j)}-\varphi\right)=\rho(f)=\infty \quad(j \in \mathbb{N}) .
$$

Now, if $\varphi(z) \equiv 0$, then we put $g(z)=f^{(j)}(z)(j \in \mathbb{N})$. It follows that

$$
\rho\left(\frac{f^{(j+1)}}{f^{(j)}}\right)=\rho\left(\frac{g^{\prime}}{g}\right)=\rho(g)=\infty,
$$

and, since $\rho_{2}(g)=\rho_{2}(f)=\rho\left(A_{0}\right)<\rho(g)=\infty$, then, by applying Lemma 18, we obtain

$$
\bar{\lambda}(g)=\lambda(g)=\infty
$$

that is, $\bar{\lambda}\left(f^{(j)}\right)=\lambda\left(f^{(j)}\right)=\infty(j \in \mathbb{N})$ and the proof of Corollary 6 is completed.

Proof of Theorem 9. By using Lemma 19, we have

$$
\begin{aligned}
g_{f} & =\frac{P_{n}[f]}{Q_{m}[f]}=\frac{\sum_{i=0}^{n} d_{i} f^{(i)}}{\sum_{j=0}^{m} b_{j} f^{(j)}} \\
& =\frac{d_{0} f+d_{1} f^{\prime}+\sum_{i=2}^{n} d_{i}\left(\alpha_{i} f^{\prime}+\beta_{i} f\right)}{b_{0} f+b_{1} f^{\prime}+\sum_{j=2}^{m} b_{j}\left(\alpha_{j} f^{\prime}+\beta_{j} f\right)},
\end{aligned}
$$

which we can write as

$$
g_{f}=\frac{P(z) f^{\prime} / f+Q(z)}{R(z) f^{\prime} / f+S(z)},
$$

where

$$
\begin{array}{ll}
P=d_{1}+\sum_{i=2}^{n} d_{i} \alpha_{i}, & Q=d_{0}+\sum_{i=2}^{n} d_{i} \beta_{i}, \\
R=b_{1}+\sum_{j=2}^{m} b_{j} \alpha_{j}, & S=b_{0}+\sum_{j=2}^{m} b_{j} \beta_{j} .
\end{array}
$$

It is clear that

$$
\max \{\rho(P), \rho(Q), \rho(R), \rho(S)\}<\rho\left(\frac{f^{\prime}}{f}\right)
$$

and, since $g_{f}$ is irreducible function in $f^{\prime} / f$, then $P S-Q R \not \equiv$ 0 . So

$$
\begin{aligned}
\rho\left(g_{f}\right) & \leq \max \left\{\rho(P), \rho(Q), \rho(R), \rho(S), \rho\left(\frac{f^{\prime}}{f}\right)\right\} \\
& =\rho\left(\frac{f^{\prime}}{f}\right) .
\end{aligned}
$$

On the other hand, we have

$$
\frac{f^{\prime}}{f}=\frac{-S(z) g_{f}+Q(z)}{R(z) g_{f}-P(z)}
$$

which implies

$$
\begin{aligned}
\rho\left(\frac{f^{\prime}}{f}\right) & \leq \max \left\{\rho(P), \rho(Q), \rho(R), \rho(S), \rho\left(g_{f}\right)\right\} \\
& =\rho\left(g_{f}\right) .
\end{aligned}
$$

By (53) and (55), we obtain $\rho\left(g_{f}\right)=\rho\left(f^{\prime} / f\right)$.

Proof of Corollary 12. Under the conditions of Corollary 12 and Lemma 22, we have $\rho(f)=\infty$ and $\rho_{2}(f)=\rho(B)$. Set

$$
g_{f}=\frac{d_{2} f^{\prime \prime}+d_{1} f^{\prime}+d_{0} f}{b_{2} f^{\prime \prime}+b_{1} f^{\prime}+b_{0} f} .
$$

Substituting $f^{\prime \prime}=-A(z) f^{\prime}-B(z) f$ into $g_{f}$, we obtain

$$
\begin{aligned}
g_{f} & =\frac{d_{2} f^{\prime \prime}+d_{1} f^{\prime}+d_{0} f}{b_{2} f^{\prime \prime}+b_{1} f^{\prime}+b_{0} f}=\frac{\left(d_{1}-d_{2} A\right) f^{\prime}+\left(d_{0}-d_{2} B\right) f}{\left(b_{1}-b_{2} A\right) f^{\prime}+\left(b_{0}-b_{2} B\right) f} \\
& =\frac{\left(d_{1}-d_{2} A\right) w+\left(d_{0}-d_{2} B\right)}{\left(b_{1}-b_{2} A\right) w+\left(b_{0}-b_{2} B\right)},
\end{aligned}
$$

where $w=f^{\prime} / f$. In order to prove that $g_{f}$ is irreducible function in $w$, we need only to prove that $h \neq \equiv 0$, where

$$
\begin{aligned}
h & =\left(d_{1}-d_{2} A\right)\left(b_{0}-b_{2} B\right)-\left(d_{0}-d_{2} B\right)\left(b_{1}-b_{2} A\right) \\
& =b_{0} d_{1}-b_{1} d_{0}+A\left(b_{2} d_{0}-b_{0} d_{2}\right)+B\left(b_{1} d_{2}-b_{2} d_{1}\right) .
\end{aligned}
$$

Since $\rho(A)<\rho(B)$ and $0<\tau(A)<\tau(B)<\infty$, if $\rho(B)=$ $\rho(A)>0, d_{2}(z) b_{1}(z)-d_{1}(z) b_{2}(z) \neq \equiv 0$, then, by Lemma 24 , we have $\rho(h)=\rho(B)>0$, so $h \not \equiv 0$. By applying Theorem 9 we obtain $\rho\left(f^{\prime} / f\right)=\rho\left(g_{f}\right)$.

Proof of Corollary 13. Suppose that $f$ is a nontrivial solution of (11) such that $\rho\left(f^{\prime} / f\right)=\infty$. Then, by Lemma 22, we have

$$
\rho(f)=+\infty, \quad \rho_{2}(f)=\rho(B) .
$$

By setting $d_{2}=b_{1} \equiv 1$ and $d_{1}=d_{0}=b_{2}=b_{0} \equiv 0$ and using Corollary 12 we have

$$
\rho\left(\frac{f^{\prime}}{f}\right)=\rho\left(\frac{f^{\prime \prime}}{f^{\prime}}\right)=\infty
$$

which implies by using Lemma 18 that

$$
\bar{\lambda}(f)=\bar{\lambda}\left(f^{\prime}\right)=\infty \text {. }
$$

\section{Conflict of Interests}

The authors declare that there is no conflict of interests regarding the publication of this paper. 


\section{References}

[1] W. K. Hayman, Meromorphic Functions, Oxford Mathematical Monographs, Clarendon Press, Oxford, UK, 1964.

[2] I. Laine, Nevanlinna theory and complex differential equations, vol. 15 of de Gruyter Studies in Mathematics, Walter de Gruyter, Berlin, Germany, 1993.

[3] B. Ya. Levin, Lectures on Entire Functions, vol. 150 of Translations of Mathematical Monographs, American Mathematical Society, Providence, RI, USA, 1996.

[4] C.-C. Yang and H.-X. Yi, Uniqueness Theory of Meromorphic Functions, vol. 557 of Mathematics and Its Applications, Kluwer Academic Publishers Group, Dordrecht, The Netherlands, 2003.

[5] A. A. Gol'dberg and V. A. Grinštel̆n, "The logarithmic derivative of a meromorphic function," Matematicheskie Zametki, vol. 19, no. 4, pp. 525-530, 1976 (Russian).

[6] D. Benbourenane and R. Korhonen, "On the growth of the logarithmic derivative," Computational Methods and Function Theory, vol. 1, no. 2, pp. 301-310, 2001.

[7] A. Hinkkanen, "A sharp form of Nevanlinna's second fundamental theorem," Inventiones Mathematicae, vol. 108, no. 3, pp. 549-574, 1992.

[8] J. Heittokangas, R. Korhonen, and J. Rättyä, "Generalized logarithmic derivative estimates of Gol'dberg-Grinshtein type," The Bulletin of the London Mathematical Society, vol. 36, no. 1, pp. 105-114, 2004.

[9] G. G. Gundersen, "Estimates for the logarithmic derivative of a meromorphic function, plus similar estimates," Journal of the London Mathematical Society, vol. 37, no. 1, pp. 88-104, 1988.

[10] Z. Latreuch and B. Belaidi, "Growth of logarithmic derivative of meromorphic functions," Mathematica Scandinavica, vol. 113, no. 2, pp. 248-261, 2013.

[11] S. B. Bank and J. K. Langley, "On the oscillation of solutions of certain linear differential equations in the complex domain," Proceedings of the Edinburgh Mathematical Society II, vol. 30, no. 3, pp. 455-469, 1987.

[12] B. Belaïdi, "The fixed points and iterated order of some differential polynomials," Commentationes Mathematicae Universitatis Carolinae, vol. 50, no. 2, pp. 209-219, 2009.

[13] I. Laine and J. Rieppo, "Differential polynomials generated by linear differential equations," Complex Variables, vol. 49, no. 12, pp. 897-911, 2004.

[14] Z. Latreuch and B. Belaïdi, "Growth and oscillation of differential polynomials generated by complex differential equations," Electronic Journal of Differential Equations, vol. 2013, no. 16, pp. 1-14, 2013.

[15] Z.-X. Chen and C.-C. Yang, "Quantitative estimations on the zeros and growths of entire solutions of linear differential equations," Complex Variables, vol. 42, no. 2, pp. 119-133, 2000.

[16] J. Tu and C.-F. Yi, "On the growth of solutions of a class of higher order linear differential equations with coefficients having the same order," Journal of Mathematical Analysis and Applications, vol. 340, no. 1, pp. 487-497, 2008.

[17] H.-Y. Xu, J. Tu, and X.-M. Zheng, "On the hyper exponent of convergence of zeros of $f^{(j)}-\varphi$ of higher order linear differential equations," Advances in Difference Equations, vol. 2012, article 114, 2012.

[18] J. Heittokangas, R. Korhonen, and J. Rättyä, "Growth estimates for solutions of linear complex differential equations," Annales Academice Scientiarum Fennicee. Mathematica, vol. 29, no. 1, pp. 233-246, 2004.
[19] T.-B. Cao, J.-F. Xu, and Z.-X. Chen, "On the meromorphic solutions of linear differential equations on the complex plane," Journal of Mathematical Analysis and Applications, vol. 364, no. 1, pp. 130-142, 2010.

[20] Z. Latreuch and B. Belaïdi, "Estimations about the order of growth and the type of meromorphic functions in the complex plane," Analele Universitatii Din Oradea. Fascicola Matematica, vol. 20, no. 1, pp. 179-186, 2013. 


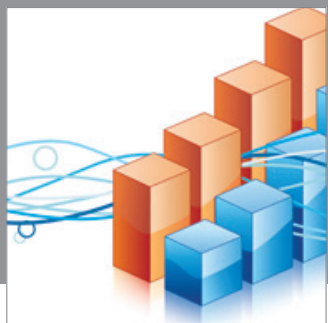

Advances in

Operations Research

mansans

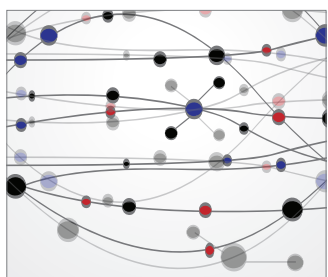

The Scientific World Journal
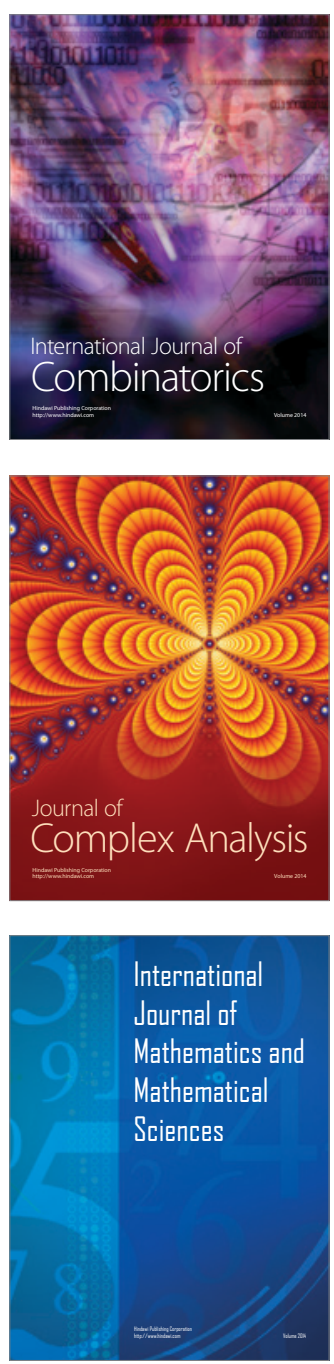
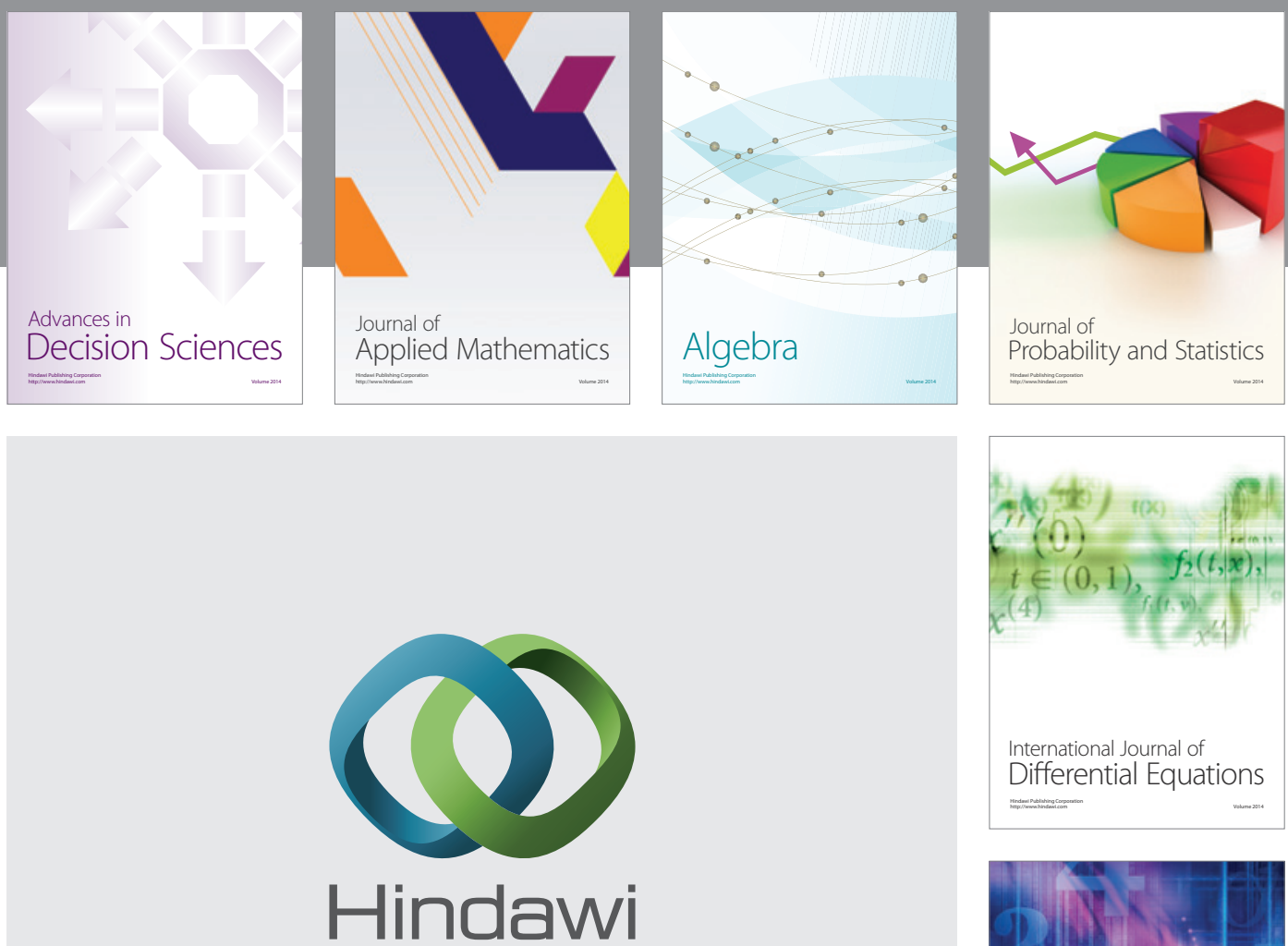

Submit your manuscripts at http://www.hindawi.com
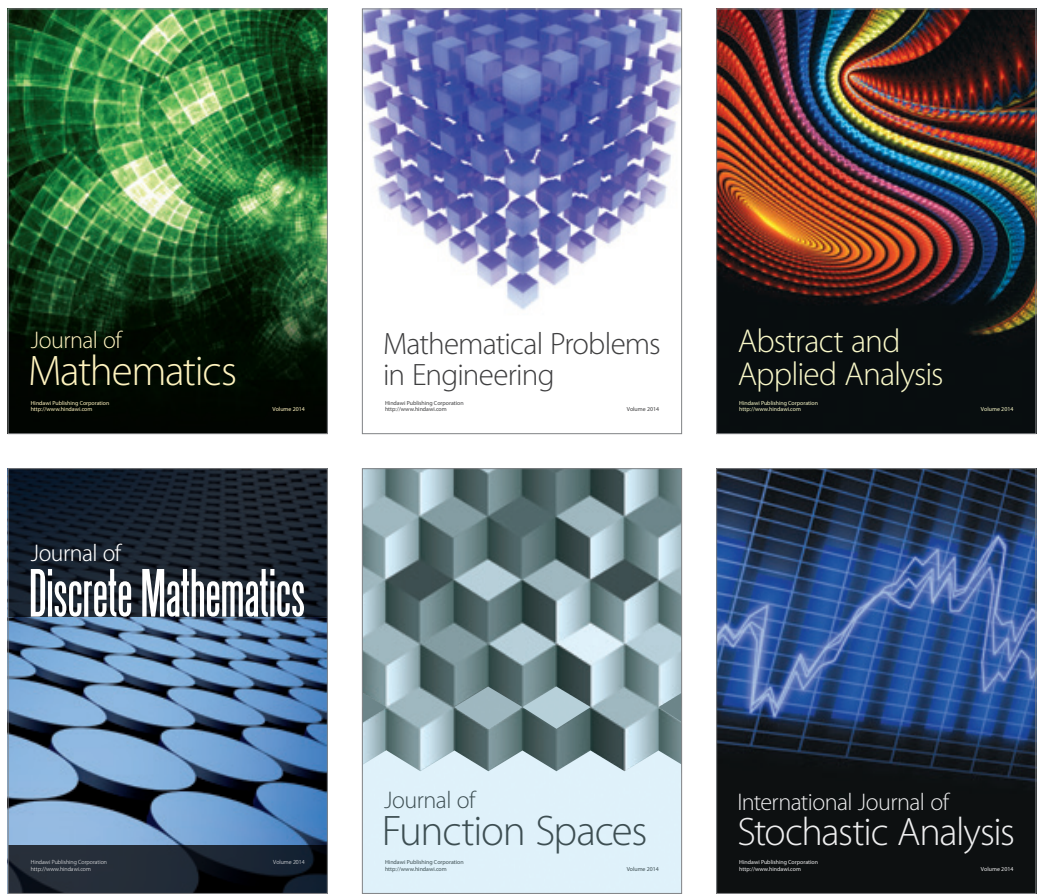

Journal of

Function Spaces

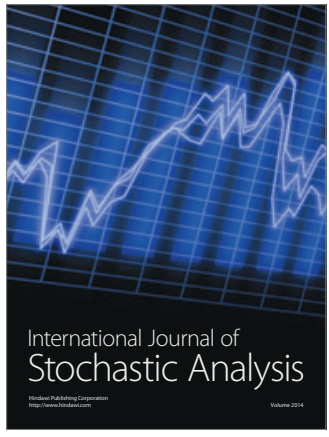

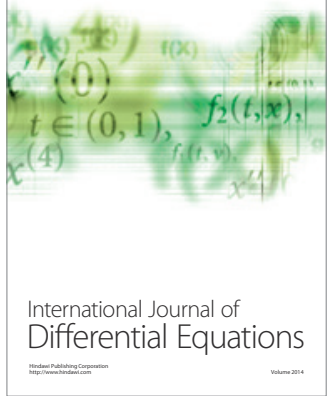
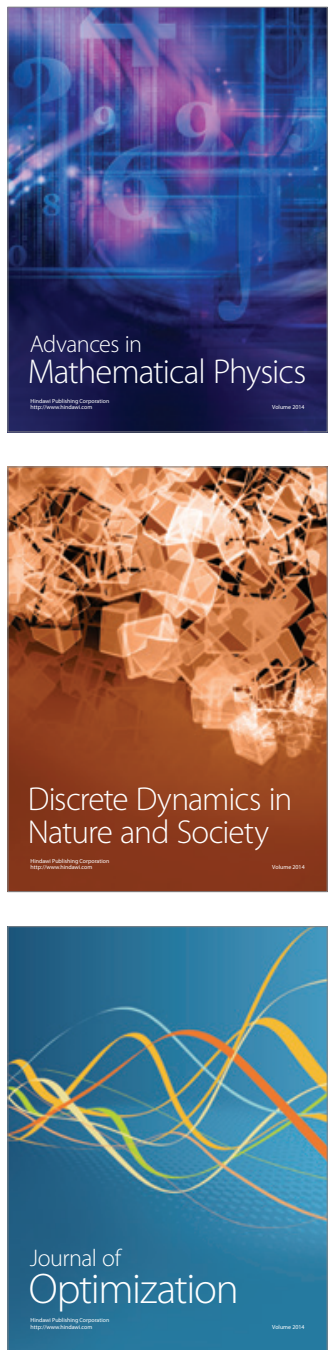